\title{
Moves and invariants for knotted handlebodies
}

\author{
Atsushi IshII
}

\begin{abstract}
We give fundamental moves for the neighborhood equivalence classes of spatial trivalent graphs. We define a coloring invariant and a cocycle invariant for the neighborhood equivalence classes and then for all spatial graphs. We show that the cocycle invariant detects the chirality of a knotted handlebody.
\end{abstract}

57M27; 57M15, 57M25

\section{Introduction}

A spatial graph is a finite graph embedded in the 3 -sphere $S^{3}$. Two spatial graphs are equivalent if there is an isotopy of $S^{3}$ taking one onto the other. S Suzuki [17] introduced the notion of the neighborhood equivalence for spatial graphs. Two spatial graphs are neighborhood equivalent if there is an isotopy of $S^{3}$ taking a regular neighborhood of one graph onto that of the other.

On the other hand, any handlebody embedded in $S^{3}$ is a regular neighborhood of some spatial trivalent graph. Hence, there is a one-to-one correspondence between the set of knotted handlebodies and that of neighborhood equivalence classes of spatial connected trivalent graphs.

It is known that two spatial trivalent graphs are equivalent if and only if their diagrams are related by a finite sequence of Reidemeister moves. However, we have not characterized such fundamental moves for the neighborhood equivalence relation. The first aim of this paper is to introduce moves for spatial trivalent graphs, called IH-moves, and show that two spatial trivalent graphs are neighborhood equivalent if and only if their diagrams are related by a finite sequence of Reidemeister moves and IH-moves (Theorem 1). It enables us to study knotted handlebodies through spatial trivalent graphs up to IH-moves.

The second aim of this paper is to define two kinds of invariants for spatial trivalent graphs. Since they are invariant under Reidemeister moves and IH-moves, we regard them as invariants for neighborhood equivalence classes of spatial (possibly nontrivalent) graphs. In fact, any spatial graph is neighborhood equivalent to some spatial trivalent graph. 
The first invariant comes from colorings for diagrams of spatial trivalent graphs (Theorem 5). Recall that the number of original Fox colorings for a diagram of a knot is an elementary invariant of the knot. It was extended to spatial Euler graphs by the author and Yasuhara [4] (see also McAtee, Silver and Williams [13]). Although a trivalent graph is not an Euler graph, we can define colorings for a spatial trivalent graph by taking enhanced constituent links of the graph. As an application, we show that there is a relationship between the coloring invariant and the tunnel number of a spatial graph (Proposition 6).

The second invariant is a generalization of the quandle cocycle invariant studied by Carter et al $[1 ; 2]$ and Satoh [16]. It is a state sum invariant defined by using a quandle coloring with a weight. We remark that Fox coloring is regarded as a coloring by the dihedral quandle; see Fenn and Rourke [3], Joyce [5], Kamada [6], Matveev [12] and Takasaki [14]. We prove that the cocycle invariant of a spatial trivalent graph is invariant under IH-moves (Theorem 7). Hence, it is equivalent to define the invariant for knotted handlebodies. In the last section (Section 6), we give an example of a knotted handlebody whose chirality is detected by the cocycle invariant. We remark that the fundamental group and then the Alexander polynomial (see Kinoshita [9;10] and Suzuki [18]) do not detect the chirality, since the complements of a spatial graph and its mirror image are homeomorphic.

\section{Moves for neighborhood equivalence classes}

Since all closed 3-manifolds have a combinatorial triangulation by Moise [15], we will work in the combinatorial category. A spatial graph is a finite graph embedded in a closed 3-manifold $M$. Two spatial graphs are equivalent if there is an isotopy of $M$ taking one onto the other. Two spatial graphs $L_{1}$ and $L_{2}$ are neighborhood equivalent if there is an isotopy of $M$ taking $N\left(L_{1}\right)$ onto $N\left(L_{2}\right)$, where $N\left(L_{1}\right)$ and $N\left(L_{2}\right)$ are respectively regular neighborhoods of $L_{1}$ and $L_{2}$. We refer the reader to Suzuki [17] for some elementary properties of the neighborhood equivalence relation.

In this paper, a trivalent graph may contain a circle component. We regard a circle as a graph without vertices. An $I H$-move is a local change of a spatial trivalent graph as described in Figure 1, where the replacement is applied in a disk embedded in $M$. Two spatial trivalent graphs are $I H$-equivalent if they are related by a finite sequence of IH-moves and isotopies of $M$. A contraction move is a local change of a spatial graph as described in Figure 1, where the replacement is applied in a disk embedded in $M$.

Theorem 1 Two spatial trivalent graphs are neighborhood equivalent if and only if they are $\mathrm{IH}$-equivalent. For any spatial graph, we obtain a spatial trivalent graph by 


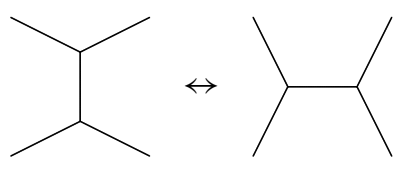

an IH-move

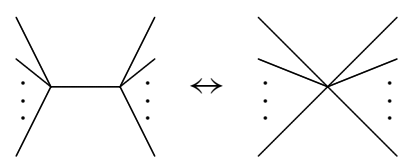

a contraction move

Figure 1

using contraction moves. This gives a one-to-one correspondence between the set of neighborhood equivalence classes of all spatial graphs and that of $\mathrm{IH}$-equivalence classes of spatial trivalent graphs.

Let $v$ be an invariant of a spatial trivalent graph. Theorem 1 implies that, if $v$ is invariant under IH-moves, then $v$ can be defined for any spatial graph and its neighborhood equivalence class.

Proof Let $N\left(L_{1}\right)$ and $N\left(L_{2}\right)$ be regular neighborhoods of spatial trivalent graphs $L_{1}$ and $L_{2}$, respectively. If $L_{1}$ and $L_{2}$ are neighborhood equivalent, there is an isotopy $\left\{f_{t}\right\}_{0 \leq t \leq 1}$ of $M$ such that

$$
f_{0}=\mathrm{id}_{M}, \quad f_{1}\left(N\left(L_{1}\right)\right)=N\left(L_{2}\right) .
$$

By Lemma 4 in the next section, $f_{1}\left(L_{1}\right)$ and $L_{2}$ are related by a finite sequence of IH-moves and isotopies. Hence, $L_{1}$ and $L_{2}$ are IH-equivalent.

We note that an IH-move does not change the isotopy class of a regular neighborhood of a spatial trivalent graph. If $L_{1}$ and $L_{2}$ are IH-equivalent, by the uniqueness of regular neighborhoods, we have an isotopy of $M$ taking $N\left(L_{1}\right)$ onto $N\left(L_{2}\right)$. Then $L_{1}$ and $L_{2}$ are neighborhood equivalent.

Since a contraction move does not change the isotopy class of a regular neighborhood of a spatial graph, the one-to-one correspondence is well defined.

We assume that $M=S^{3}$. Any two diagrams of equivalent spatial graphs are related by a finite sequence of the moves R1-3, N4-5 in Figure 2 [7; 20]. Since we may apply an IH-move in a small disk by an isotopy of $S^{3}$, we have the following corollary.

Corollary 2 Let $D_{1}$ and $D_{2}$ be diagrams of spatial trivalent graphs $L_{1}$ and $L_{2}$, respectively. Then $L_{1}$ and $L_{2}$ are neighborhood equivalent if and only if $D_{1}$ and $D_{2}$ are related by a finite sequence of the moves R1-6 depicted in Figure 2. 

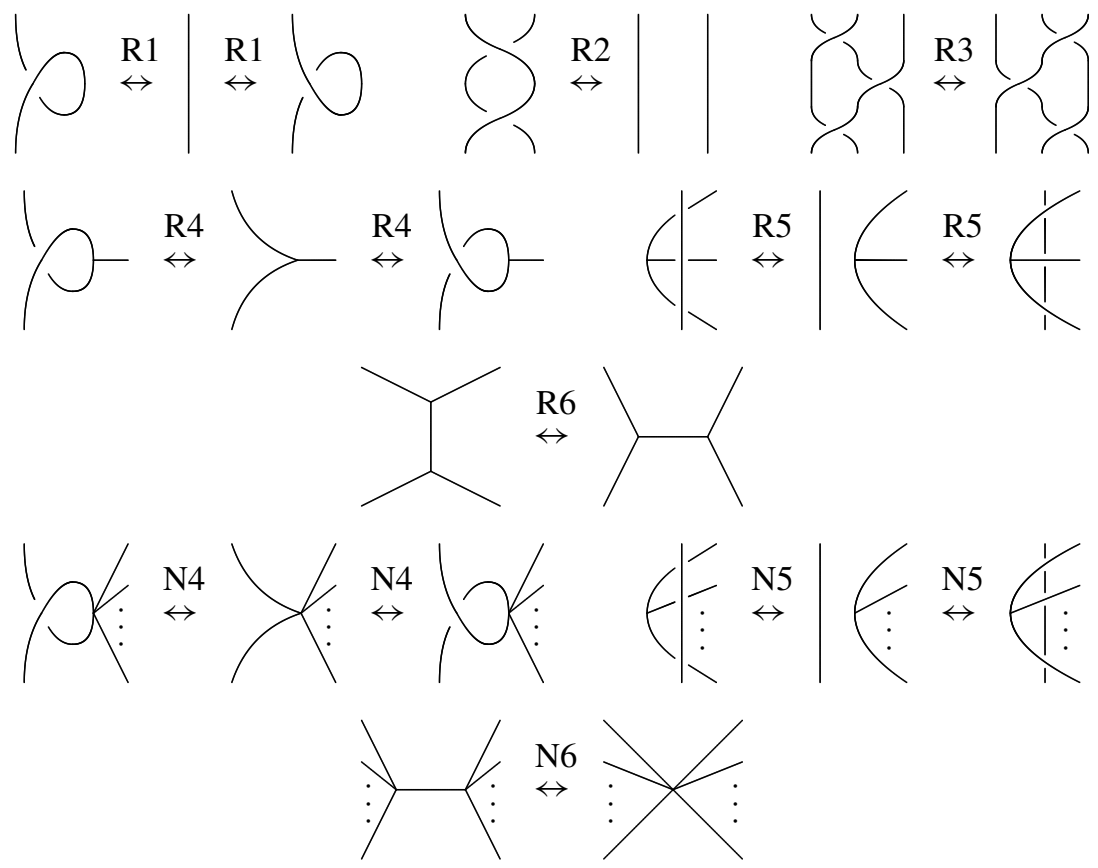

Figure 2

Two spatial graphs are neighborhood equivalent if and only if they are related by a finite sequence of contraction moves and isotopies of $S^{3}$, since an IH-move can be realized by contraction moves. Since we may apply a contraction move in a small disk by an isotopy of $S^{3}$, we have the following corollary.

Corollary 3 Let $D_{1}$ and $D_{2}$ be diagrams of spatial graphs $L_{1}$ and $L_{2}$, respectively. Then $L_{1}$ and $L_{2}$ are neighborhood equivalent if and only if $D_{1}$ and $D_{2}$ are related by a finite sequence of the moves R1-R3, N4-N6 depicted in Figure 2.

By Corollary 2, we can study a handlebody embedded in $S^{3}$ through a diagram, since it can be obtained from a regular neighborhood of a spatial trivalent graph. We prepare terminologies for this study below. A handlebody-link diagram is a spatial trivalent graph diagram, where a trivalent graph may contain circle components. Two handlebody-link diagrams are said to be equivalent if they are related by a finite sequence of the moves R1-6 depicted in Figure 2. A handlebody-link is the equivalence class of a handlebody-link diagram. A handlebody-knot is a handlebody-link with one component. By Corollary 2, a handlebody-link corresponds to handlebodies embedded in $S^{3}$. 


\section{A spine of a handlebody}

Let $M$ be a closed 3-manifold. Let $H$ be a handlebody embedded in $M$. We assume that the handlebody $H$ is of genus $g>1$. A trivalent spine of $H$ is a trivalent graph $K$ embedded in $H$ such that a regular neighborhood of $K$ is $H$.

Lemma 4 Any two trivalent spines $K$ and $K^{\prime}$ of $H$ are related by a finite sequence of IH-moves and isotopies.

Let $\left\{D_{j}\right\}$ be properly embedded mutually disjoint disks in $H$. We denote by $B\left(\left\{D_{j}\right\}\right)$ the set of connected components obtained by cutting $H$ along the disks $\left\{D_{j}\right\}$. If $B\left(\left\{D_{j}\right\}\right)$ is the set of 3 -balls, we call the disks $\left\{D_{j}\right\}$ a system of disks for $H$. A system of disks $\left\{D_{j}\right\}$ is maximal if each 3 -ball in $B\left(\left\{D_{j}\right\}\right)$ has three disks in its boundary. Then there is a one-to-one correspondence between the set of trivalent spines and that of maximal systems of disks up to isotopy: For each trivalent spine, there is a unique maximal system of disks such that the spine is dual to the disks.

For a proof of Lemma 4, we keep a trivalent spine and a maximal system of disks all together. A sd-system of $H$ is a pair of a trivalent spine $K$ of $H$ and a maximal system of disks $\left\{D_{j}\right\}$ for $H$ such that $K$ appears in each 3-ball in $B\left(\left\{D_{j}\right\}\right)$ as a trivial Y-tangle, where a trivial Y-tangle is a $\mathrm{Y}$-shaped graph properly embedded in a 3-ball which is homeomorphic to the 3-ball shown in Figure 3. We note that, if $T$ and $T^{\prime}$ are trivial Y-tangles with the same endpoints, then there is an isotopy of the 3-ball taking $T$ onto $T^{\prime}$ relative to the boundary.

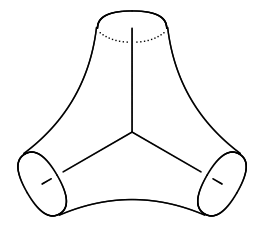

Figure 3

Proof of Lemma 4 A marking

$$
m=\bigcup_{j=1}^{3 g-3} m_{j}
$$

is a disjoint union of $3 g-3$ pairwise nonparallel, essential unoriented simple loops in $\partial H$. For the trivalent spines $K$ and $K^{\prime}$, there are sd-systems $\left(K,\left\{D_{j}\right\}\right)$ and 
$\left(K^{\prime},\left\{D_{j}^{\prime}\right\}\right)$, respectively. FLuo [11, Corollary 1] showed that there exists a finite sequence of markings

$$
\begin{gathered}
m^{i}=\bigcup_{j=1}^{3 g-3} m_{j}^{i} \quad(i=1, \ldots, n) \\
m^{1}=\bigcup_{j=1}^{3 g-3} \partial D_{j} \quad \text { and } \quad m^{n}=\bigcup_{j=1}^{3 g-3} \partial D_{j}^{\prime},
\end{gathered}
$$

such that

and such that $m_{j}^{i}=m_{j}^{i+1}$ for $j \neq k_{i}$ and $m_{k_{i}}^{i} \cap m_{k_{i}}^{i+1}$ consists of two points of different intersection signs as shown in Figure 4. By reordering the disks if necessary, we may assume that $m_{j}^{1}=\partial D_{j}$ and $m_{j}^{n}=\partial D_{j}^{\prime}$ for any $j$.
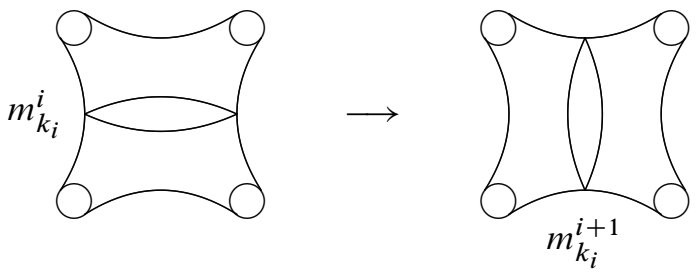

Figure 4

For $i=1, \ldots, n$, we take a sd-system $\left(K^{i},\left\{D_{j}^{i}\right\}\right)$ such that $\partial D_{j}^{i}=m_{j}^{i}$ for any $j$ as follows. Set $K^{1}:=K, D_{j}^{1}:=D_{j}$ for any $j$. Since the spine $K^{1}$ appears in each 3-ball in $B\left(\left\{D_{j}^{1}\right\}\right)$ as a trivial Y-tangle, we may position the disk $D_{k_{1}}^{1}$, the spine $K^{1}$, and the marking $m_{k_{1}}^{2}$ as depicted in the left diagram of Figure 5. Replacing $K^{1}$ and $D_{k_{1}}^{1}$ by the spine and the disk depicted in the right diagram, we obtain a sd-system $\left(K^{2},\left\{D_{j}^{2}\right\}\right)$ such that $\partial D_{k_{1}}^{2}=m_{k_{1}}^{2}$ and $D_{j}^{2}=D_{j}^{1}$ for $j \neq k_{1}$. We remark that $K^{2}$ is obtained from $K^{1}$ by an IH-move and isotopies. Repeating this procedure, we obtain $\left(K^{i},\left\{D_{j}^{i}\right\}\right)$ for $i=1, \ldots, n$.
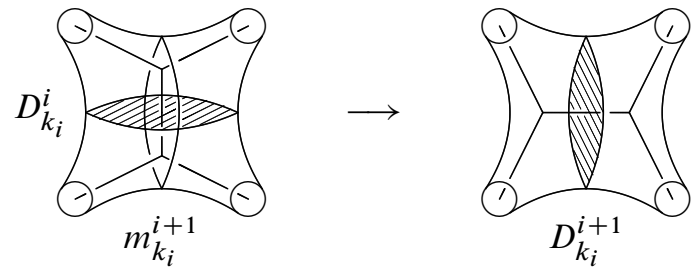

Figure 5

Since a handlebody is irreducible, there is an isotopy $\left\{f_{t}\right\}_{0 \leq t \leq 1}$ of $H$ such that

$$
f_{0}=\operatorname{id}_{H}, \quad f_{1}\left(D_{j}^{n}\right)=D_{j}^{\prime}, \quad f_{1}\left(D_{j}^{n} \cap K^{n}\right)=D_{j}^{\prime} \cap K^{\prime},
$$


for any $j$. Since the spine $f_{1}\left(K^{n}\right)$ and $K^{\prime}$ appear in each 3-ball in $B\left(\left\{D_{j}^{\prime}\right\}\right)$ as trivial Y-tangles, there is an isotopy of $H$ taking $f_{1}\left(K^{n}\right)$ onto $K^{\prime}$. Therefore $K$ and $K^{\prime}$ are related by a finite sequence of $\mathrm{IH}$-moves and isotopies.

\section{Enhanced constituent links and colorings}

Let $L$ be a spatial trivalent graph. Let $\rho$ be a map from the set of edges of $L$ to $\mathbb{Z} / 2 \mathbb{Z}$ such that $\rho\left(e_{1}\right)+\rho\left(e_{2}\right)+\rho\left(e_{3}\right)=0$ for edges $e_{1}, e_{2}, e_{3}$ incident to a vertex. We denote by $\mathcal{R}(L)$ the set of such maps. For a spatial trivalent graph $L$ and $\rho \in \mathcal{R}(L)$, we call the pair $(L, \rho)$ an enhanced constituent link of $L$. The spatial trivalent graph depicted in the left diagram of Figure 6 has four enhanced constituent links, where edges assigned 1 by the map $\rho$ are drawn thickly. We call $\rho(e)$ the reality of an edge $e$. We remark that, for an enhanced constituent link $(L, \rho)$, edges whose realities are 1 form a constituent link of $L$. Let $E$ be a diagram of an enhanced constituent link $(L, \rho)$. We denote by $\mathcal{A}(E)$ the set of the arcs of $E$, where an arc is a piece of a curve such that its endpoint is an undercrossing or a vertex. The reality of an arc is defined by that of an edge such that the arc originates from the edge, which is denoted by using the same symbol $\rho$.
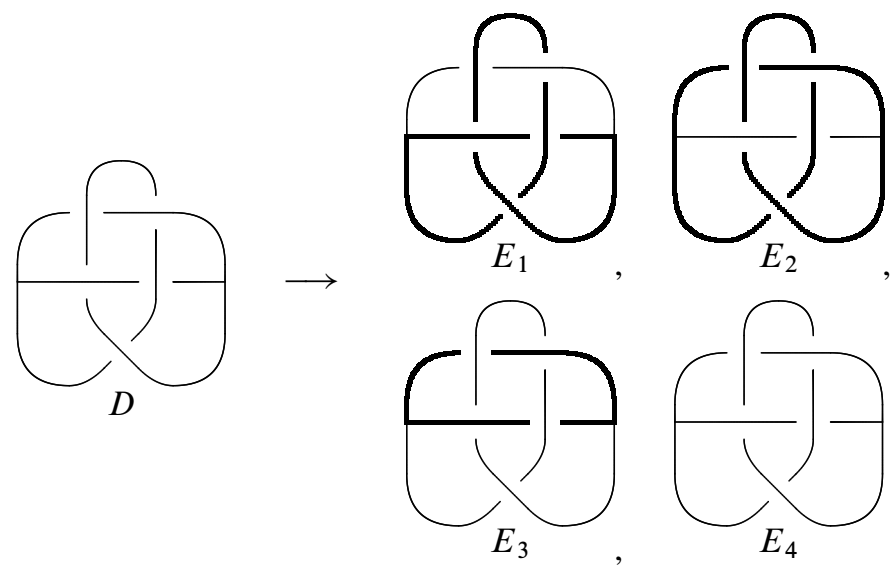

Figure 6

A $k e i[5 ; 6 ; 14]$ is a nonempty set $X$ with a binary operation $(a, b) \mapsto a * b$ satisfying the following axioms:

(K1) For any $a \in X, a * a=a$.

(K2) For any $a, b \in X,(a * b) * b=a$.

(K3) For any $a, b, c \in X,(a * b) * c=(a * c) *(b * c)$. 
In this paper, we suppose that a kei is finite. Set $a *^{0} b:=a$ and $a *^{1} b:=a * b$. A map $C: \mathcal{A}(E) \rightarrow X$ is an $X$-coloring of a diagram $E$ representing an enhanced constituent link $(L, \rho)$ if

$$
\begin{array}{ll}
C\left(\chi_{1}\right) *^{\rho\left(\chi^{1}\right)} C\left(\chi^{1}\right)=C\left(\chi_{2}\right) & \text { at each crossing } \chi, \\
C\left(\omega_{1}\right)=C\left(\omega_{2}\right)=C\left(\omega_{3}\right) & \text { at each vertex } \omega,
\end{array}
$$

where $\chi_{1}, \chi_{2}$ and $\chi^{1}$ are the under-arcs and the over-arc at a crossing $\chi$, and $\omega_{1}, \omega_{2}, \omega_{3}$ are the arcs incident to a vertex $\omega$. See Figure 7, where an underline indicates the reality of an arc. The axiom (K2) implies that the $X$-coloring is well defined. We call $C(\alpha)$ the color of an arc $\alpha$. We denote by $\operatorname{Col}_{X}(E)$ the set of $X$-colorings of $E$.
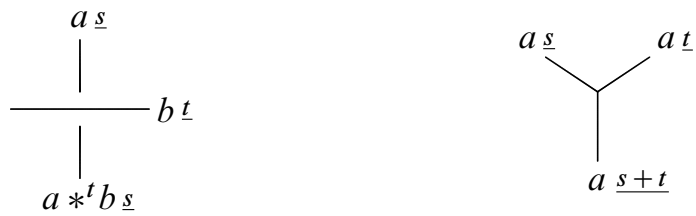

Figure 7

We denote by $E C(D)$ the set of enhanced constituent link diagrams obtained from a spatial trivalent graph diagram $D$, and denote by $\# A$ the number of elements in a set $A$.

Theorem 5 Let $D$ be a diagram of a spatial trivalent graph $L$. Let $D^{\prime}$ be a diagram obtained by applying one of the moves R1-R6 to $D$ once. Then we have the following.

- For $E \in E C(D)$, there is a unique enhanced constituent link diagram $E_{D, D^{\prime}} \in$ $E C\left(D^{\prime}\right)$ such that the realities are preserved in the outside of the neighborhood in which the move is applied: There is a one-to-one correspondence between $E C(D)$ and $E C\left(D^{\prime}\right)$.

- For $C \in \operatorname{Col}_{X}(E)$, there is a unique $X$-coloring $C_{D, D^{\prime}} \in \operatorname{Col}_{X}\left(E_{D, D^{\prime}}\right)$ such that the colors are preserved in the outside of the neighborhood in which the move is applied: There is a one-to-one correspondence between $\operatorname{Col}_{X}(E)$ and $\operatorname{Col}_{X}\left(E_{D, D^{\prime}}\right)$.

Thus, for a finite kei $X$, the multiset $\left\{\# \operatorname{Col}_{X}(E) \mid E \in E C(D)\right\}$ is an invariant for any spatial graph obtained from $L$ by contraction moves, and for its neighborhood equivalence class.

We note that the multiset is an invariant for a handlebody-link. 
Proof The one-to-one correspondences are obtained from Figure 8. They are well defined, since we have the following equalities:

$\left(\mathrm{K} 1^{\prime}\right)$ For any $a \in X$ and $s \in \mathbb{Z} / 2 \mathbb{Z}, a *^{s} a=a$.

$\left(\mathrm{K} 2^{\prime}\right)$ For any $a, b \in X$ and $s \in \mathbb{Z} / 2 \mathbb{Z},\left(a *^{s} b\right) *^{s} b=a$.

$\left(\mathrm{K}^{\prime}\right)$ For any $a, b, c \in X$ and $s, t \in \mathbb{Z} / 2 \mathbb{Z},\left(a *^{s} b\right) *^{t} c=\left(a *^{t} c\right) *^{s}\left(b *^{t} c\right)$.

$\left(\mathrm{K} 4^{\prime}\right)$ For any $a, b \in X$ and $s, t \in \mathbb{Z} / 2 \mathbb{Z}, a *^{(s+t)} b=\left(a *^{s} b\right) *^{t} b$.

Then the multiset is an invariant of a spatial trivalent graph $L$, which is invariant under IH-moves.

\section{Colorings by the dihedral kei}

The dihedral kei of order $p$, denoted by $R_{p}$, is a kei consisting of the set $\mathbb{Z} / p \mathbb{Z}$ with the binary operation defined by $a * b=2 b-a$. We suppose that $p$ is an odd prime. Let $E$ be an enhanced constituent link diagram. The coloring system of $E$ is the system of linear equations obtained from the coloring relations at crossings and vertices of $E$. The set $\operatorname{Col}_{R_{p}}(E)$ is a vector space over $\mathbb{Z} / p \mathbb{Z}$, since it is a solution space of the coloring system of $E$. Then, for a spatial trivalent graph $L$ represented by a diagram $D$, we define the polynomial $f_{p}(L ; t)=\sum_{E \in E C(D)} t^{m(E)}$, where $m(E)$ is the integer satisfying $\operatorname{Col}_{R_{p}}(E) \cong(\mathbb{Z} / p \mathbb{Z})^{m(E)+1}$. By Theorem $5, f_{p}(L ; t)$ is an invariant of $L$, and its neighborhood equivalence class. Then $f_{p}(L ; t)$ is defined for any spatial graph $L$.

A spatial graph is neighborhood trivial if it is neighborhood equivalent to a spatial graph embedded in a plane. The spatial trivalent graph $K$ represented by the diagram $D$ of Figure 6 is knotted, since a constituent link of $K$ is the trefoil knot. However this does not imply that $K$ is not neighborhood trivial.

We evaluate the invariant $f_{3}(K ; t)$, and show that $K$ is not neighborhood trivial. For an enhanced constituent link diagram in Figure 6, let $a_{l}$ (resp. $a_{r}$ ) be the color of arcs incident to the left (resp. right) vertex, and let $a_{t}$ be the color of the top arc, and let $a_{b}$ be the color of the other arc. The coloring system of $E_{1}$ is

$$
\begin{aligned}
& 2 a_{t}-a_{l}-a_{r}=0, \quad a_{t}-a_{b}=0, \quad 2 a_{l}-a_{t}-a_{r}=0, \quad 2 a_{b}-a_{l}-a_{r}=0, \\
& 2 a_{r}-a_{l}-a_{b}=0 .
\end{aligned}
$$



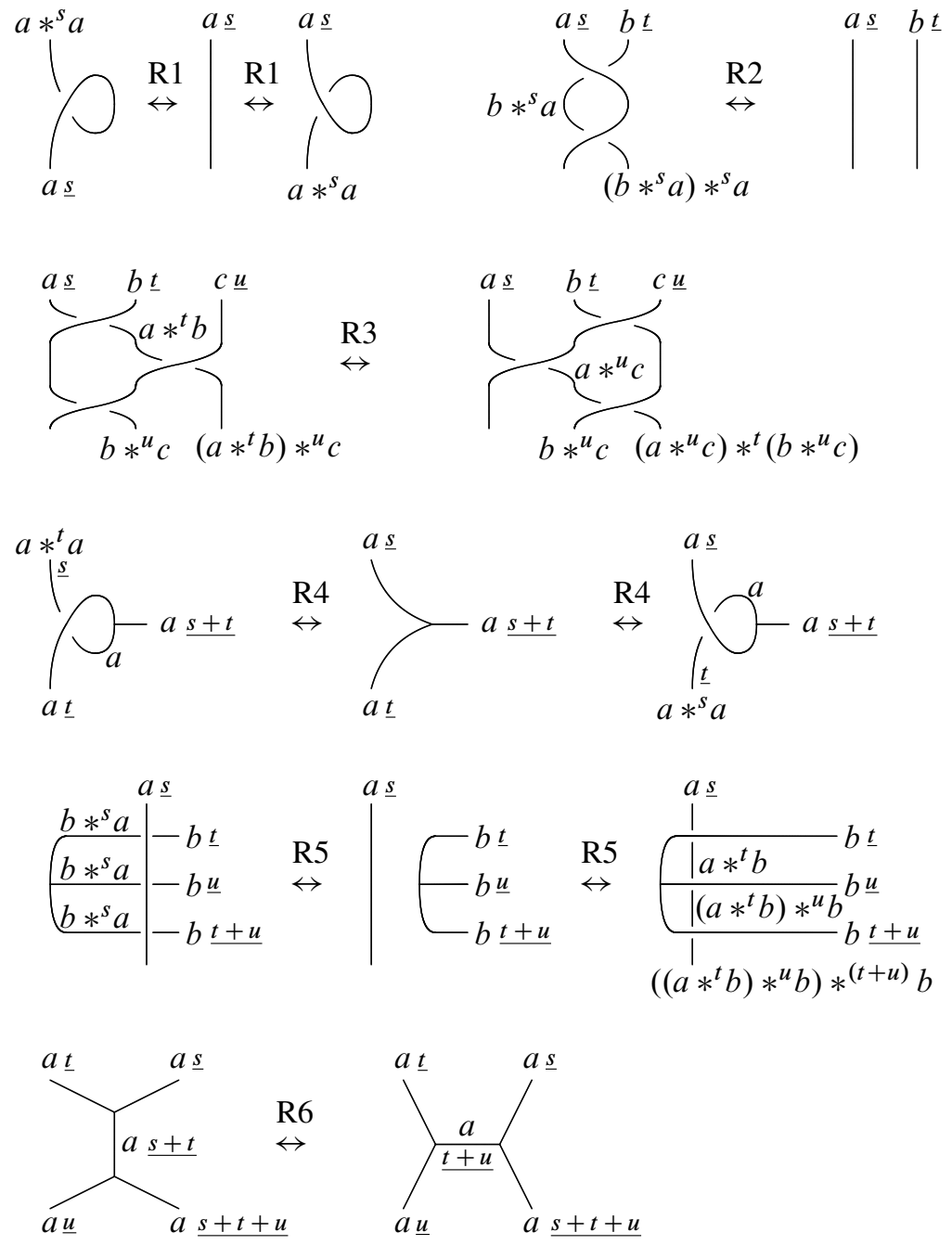

Figure 8

Then we have the following $(5,4)$-matrix over $\mathbb{Z} / 3 \mathbb{Z}$ representing this system:

$$
A_{1}:=\left(\begin{array}{cccc}
-1 & -1 & 2 & 0 \\
0 & 0 & 1 & -1 \\
2 & -1 & -1 & 0 \\
-1 & -1 & 0 & 2 \\
-1 & 2 & 0 & -1
\end{array}\right)
$$


Since $\operatorname{rank} A_{1}=2$, we have $\operatorname{Col}_{R_{3}}\left(E_{1}\right) \cong(\mathbb{Z} / 3 \mathbb{Z})^{2}$. Let $A_{i}$ be the matrix over $\mathbb{Z} / 3 \mathbb{Z}$ representing the coloring system of the enhanced constituent link diagram $E_{i}$ for $i=2,3,4$. Since the matrices $A_{2}, A_{3}, A_{4}$ are respectively given by

$$
\left(\begin{array}{cccc}
-1 & -1 & 2 & 0 \\
0 & 2 & -1 & -1 \\
0 & -1 & 1 & 0 \\
-1 & -1 & 0 & 2 \\
-1 & 2 & 0 & -1
\end{array}\right), \quad\left(\begin{array}{cccc}
1 & -1 & 0 & 0 \\
0 & 2 & -1 & -1 \\
2 & -1 & -1 & 0 \\
1 & -1 & 0 & 0 \\
-1 & 0 & 0 & 1
\end{array}\right),\left(\begin{array}{cccc}
1 & -1 & 0 & 0 \\
0 & 0 & 1 & -1 \\
0 & -1 & 1 & 0 \\
1 & -1 & 0 & 0 \\
-1 & 0 & 0 & 1
\end{array}\right),
$$

we have $\operatorname{rank} A_{i}=3$ and then $\operatorname{Col}_{R_{3}}\left(E_{i}\right) \cong \mathbb{Z} / 3 \mathbb{Z}$ for $i=2,3,4$. Thus we have

$$
f_{3}(K ; t)=t+3 \text {. }
$$

On the other hand, we have

$$
f_{p}\left(O_{g} ; t\right)=2^{g},
$$

where $O_{g}$ is the spatial graph depicted in Figure 9. Then $K$ is not neighborhood trivial, since any neighborhood trivial spatial connected graph is neighborhood equivalent to $O_{g}$ for some $g$.

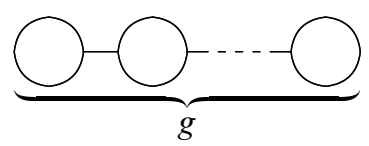

Figure 9

The tunnel number $t(L)$ of a spatial graph $L$ is the minimum number of disjoint properly embedded edges in the exterior of $L$ such that the edges and $L$ form a neighborhood trivial spatial connected graph (see Kawauchi [8, p 207]). We remark that, a spatial graph is connected and neighborhood trivial if and only if the complement of a neighborhood of the spatial graph is a handlebody, since there is only one Heegaard splitting of $S^{3}$ for each genus [19].

Proposition 6 For a spatial graph $L$, we have

$$
t(L) \geq \operatorname{deg} f_{p}(L ; t) .
$$

Proof For a spatial graph $L$, we have a sequence $L=L_{0}, \ldots, L_{t(L)}=O$ such that $L_{i+1}$ is a spatial graph obtained by attaching an edge to $L_{i}$, where $O$ is a neighborhood trivial spatial connected graph. If two spatial graphs $M_{1}$ and $M_{2}$ are neighborhood equivalent, then $t\left(M_{1}\right)=t\left(M_{2}\right)$ and $f_{p}\left(M_{1} ; t\right)=f_{p}\left(M_{2} ; t\right)$. Hence we assume that $L_{0}, \ldots, L_{t(L)}$ are spatial trivalent graphs. 
The spatial trivalent graphs $L_{i}$ and $L_{i+1}$ respectively have diagrams $D_{i}$ and $D_{i}^{\prime}$ which are identical except in the neighborhood of a point where they differ as shown in Figure 10. Let $E_{i}$ be an enhanced constituent link diagram of $D_{i}$ such that

$$
\operatorname{Col}_{R_{p}}\left(E_{i}\right) \cong(\mathbb{Z} / p \mathbb{Z})^{\operatorname{deg} f_{p}\left(L_{i} ; t\right)+1} .
$$

There is a unique enhanced constituent link diagram $E_{i}^{\prime} \in E C\left(D_{i}^{\prime}\right)$ which is obtained by attaching an edge of reality 0 to $E_{i}$. Since the coloring system of $E_{i}^{\prime}$ is obtained from that of $E_{i}$ by adding one relation, we have

$$
\operatorname{Col}_{R_{p}}\left(E_{i}^{\prime}\right) \cong(\mathbb{Z} / p \mathbb{Z})^{\operatorname{deg} f_{p}\left(L_{i} ; t\right)} \quad \text { or } \quad(\mathbb{Z} / p \mathbb{Z})^{\operatorname{deg} f_{p}\left(L_{i} ; t\right)+1} .
$$

Hence, we have

$$
\operatorname{deg} f_{p}\left(L_{i+1} ; t\right) \geq \operatorname{deg} f_{p}\left(L_{i} ; t\right)-1,
$$

which implies

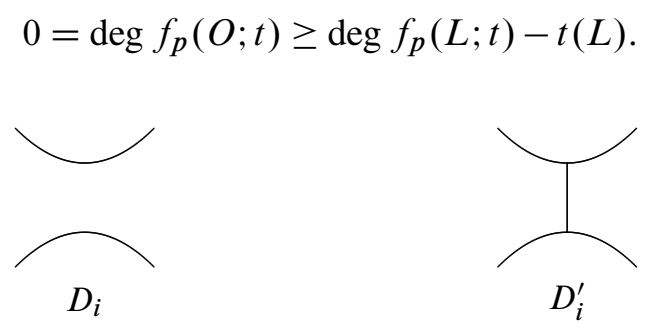

Figure 10

Since the tunnel number of a spatial graph depends on its neighborhood equivalence class, the tunnel number is defined for a handlebody-link, and the same inequality in Proposition 6 holds for handlebody-links.

\section{A cocycle invariant}

Let $E$ be a diagram of an enhanced constituent link $(L, \rho)$. We denote by $\widetilde{\mathcal{A}}(E)$ the union of $\mathcal{A}(E)$ and the set of connected regions of the complement of the underlying immersed graph of $E$. A map $C: \widetilde{\mathcal{A}}(E) \rightarrow X$ is a shadow $X$-coloring of $E$ if $\left.C\right|_{\mathcal{A}(E)} \in \operatorname{Col}_{X}(E)$ and

$$
C\left(r_{1}\right) *^{\rho(\alpha)} C(\alpha)=C\left(r_{2}\right),
$$

where $r_{1}, r_{2}$ are regions sharing an arc $\alpha$. See the left diagram of Figure 11, where a rectangle indicates the color of a region. The equalities $\left(\mathrm{K} 2^{\prime}\right)-\left(\mathrm{K} 4^{\prime}\right)$ imply that the shadow $X$-coloring is well defined: $\left(\mathrm{K} 2^{\prime}\right)$ at an arc, $\left(\mathrm{K}^{\prime}\right)$ at a crossing, and $\left(\mathrm{K} 4^{\prime}\right)$ at a vertex. We denote by $\widetilde{\mathrm{Col}_{X}}(E)$ the set of shadow $X$-colorings of $E$. 

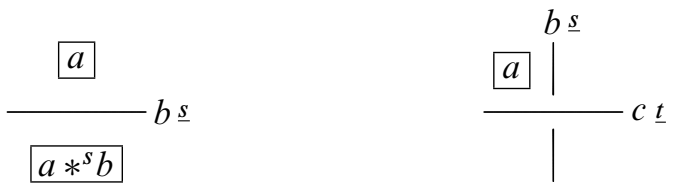

Figure 11

Let $\theta(x, y, z) \in \mathbb{Z}[x, y, z]$ be the polynomial determined by

$$
(x-y) \frac{(2 z-y)^{p}+y^{p}-2 z^{p}}{p},
$$

where we remark that the numerator is divisible by $p$. Put

$$
\theta_{p}(a, b, c)=\theta(a, b, c) \bmod p,
$$

which is Mochizuki's 3-cocycle. For $C \in \widetilde{\mathrm{Col}}_{R_{p}}(E)$, the Boltzmann weight $B(\chi ; C) \in$ $\mathbb{Z} / p \mathbb{Z}$ at a crossing $\chi$ is defined by

$$
B(\chi ; C)=\theta_{p}(a, b, c),
$$

where $a, b, c$ are the colors of a region, an under-arc, and the over-arc at the crossing $\chi$ as shown in the right diagram of Figure 11. The Boltzmann weight does not depend on the choice of the under-arc (and the region), since we have the equality

$$
\theta_{p}(a, b, c)=\theta_{p}(2 c-2 b+a, 2 c-b, c) .
$$

A crossing of an enhanced constituent link diagram is said to be real if the crossing consists of three arcs of reality 1 . For $C \in \widetilde{\mathrm{Col}}_{R_{p}}(E)$, set

$$
B(C):=\sum_{\chi} B(\chi ; C),
$$

where $\chi$ runs over all real crossings. Then, for a spatial trivalent graph $L$ represented by a diagram $D$, we define the $p$-variable polynomial

$$
\Phi_{p}\left(D ; x_{0}, x_{1}, \ldots, x_{p-1}\right)=\sum_{E \in E C(D)} \prod_{C \in \widetilde{\operatorname{Col}}_{R_{p}}(E)} x_{B(C)} .
$$

Theorem 7 Let $D$ be a diagram of a spatial trivalent graph $L$. The $p$-variable polynomial $\Phi_{p}\left(D ; x_{0}, x_{1}, \ldots, x_{p-1}\right)$ is an invariant for any spatial graph obtained from $L$ by contraction moves, and for its neighborhood equivalence class.

By this theorem, we denote by $\Phi_{p}\left(L ; x_{0}, x_{1}, \ldots, x_{p-1}\right)$ the $p$-variable polynomial of $D$. We note that the $p$-variable polynomial is an invariant for a handlebody-link. 
Proof Let $D^{\prime}$ be a diagram obtained by applying one of the moves R1-R6 to $D$ once. As in the case of $X$-colorings, for $E \in E C(D)$ and $C \in \widetilde{\mathrm{Col}}_{R_{p}}(E)$, there is a unique shadow $R_{p}$-coloring $C_{D, D^{\prime}} \in \widetilde{\mathrm{Col}}_{R_{p}}\left(E_{D, D^{\prime}}\right)$ such that the colors are preserved in the outside of the neighborhood in which the move is applied. It is sufficient to show that $B(C)=B\left(C_{D, D^{\prime}}\right)$.

Let $x$ be the color of the leftmost region in each diagram of Figure 8. For the moves $\mathrm{R} 1$ and $\mathrm{R} 4$, the equality $B(C)=B\left(C_{D, D^{\prime}}\right)$ follows from the equality

$$
\theta_{p}(x, a, a)=0 \text {. }
$$

For the moves R2 and R5, the equality $B(C)=B\left(C_{D, D^{\prime}}\right)$ follows from the equality

$$
\theta_{p}(2 a-x, b, a)+\theta_{p}(x, 2 a-b, a)=0 .
$$

For the move R3, the equality $B(C)=B\left(C_{D, D^{\prime}}\right)$ follows from the equality

$$
\begin{aligned}
\theta_{p}(x, a, b)+\theta_{p}(2 b-x, & 2 b-a, c)+\theta_{p}(x, b, c) \\
= & \theta_{p}(2 a-x, b, c)+\theta_{p}(x, a, c)+\theta_{p}(2 c-x, 2 c-a, 2 c-b),
\end{aligned}
$$

that is the cocycle condition of $\theta_{p}$. Therefore $\Phi_{p}\left(D ; x_{0}, x_{1}, \ldots, x_{p-1}\right)$ is an invariant of $L$.

We denote by $L^{*}$ the mirror image of a spatial graph $L$. Then we have the following proposition.

Proposition 8 For a spatial graph $L$, we have

$$
\Phi_{p}\left(L^{*} ; x_{0}, x_{1}, \ldots, x_{p-1}\right)=\Phi_{p}\left(L ; x_{0}, x_{-1}, \ldots, x_{-(p-1)}\right),
$$

where we note that $x_{-i}=x_{n-i}$.

Proof Let $D$ be a diagram of $L$. We suppose that $D$ is depicted in an $x y$-plane. Let $\varphi$ be the involution $(x, y) \mapsto(-x, y)$. For $E \in E C(D)$ and $C \in \widetilde{\mathrm{Col}}_{R_{p}}(E)$, we have $\varphi(E) \in E C(\varphi(D))$ and $C \circ \varphi \in{\widetilde{\mathrm{Col}_{R}}}_{R_{p}}(\varphi(E))$, where the reality of an $\operatorname{arc} \alpha$ of $\varphi(E)$ is given by that of the $\operatorname{arc} \varphi(\alpha)$ of $E \in E C(D)$. By the equality $\theta_{p}(a, b, c)=-\theta_{p}(2 b-a, b, c)$, we have $B(\chi, C)=-B(\varphi(\chi), C \circ \varphi)$ for each crossing $\chi$ of $D$ (see Figure 12). Thus, we have the equality

$$
\Phi_{p}\left(L^{*} ; x_{0}, x_{1}, \ldots, x_{p-1}\right)=\Phi_{p}\left(L ; x_{0}, x_{-1}, \ldots, x_{-(p-1)}\right) .
$$




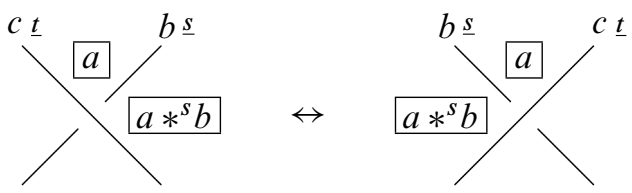

Figure 12

A spatial graph $L$ is chiral if $L$ and $L^{*}$ are not equivalent. Let $K$ be the spatial trivalent graph represented by the diagram $D$ of Figure 6 . Then we have

$$
\begin{gathered}
\Phi_{3}\left(K ; x_{0}, x_{1}, x_{2}\right)=x_{0}^{9} x_{2}^{18}+3 x_{0}^{9}, \\
\Phi_{3}\left(K^{*} ; x_{0}, x_{1}, x_{2}\right)=x_{0}^{9} x_{1}^{18}+3 x_{0}^{9} .
\end{gathered}
$$

This implies not only that $K$ is chiral as a spatial graph but also that $K$ is chiral as a handlebody-link. We can check the chirality of $K$ as a spatial graph without invariants, since the only nontrivial constituent link of $K$ is the trefoil knot, that is chiral. However it is not easy to check the chirality of a knotted handlebody, since invariants derived from its complement does not work. The invariant $\Phi_{p}$ is the first polynomial invariant which detects the chirality of a knotted handlebody.

Acknowledgments The author would like to thank Masahide Iwakiri, Akio Kawauchi, Kazuyuki Kuwako, Hiromasa Moriuchi, Takuji Nakamura, Yasutaka Nakanishi, Makoto Sakuma, Shin Satoh, Shinichi Suzuki, Kouki Taniyama, and Ryosuke Yamamoto for their helpful comments. This research is partially supported by Grant-in-Aid for JSPS Research Fellowships for Young Scientists.

\section{References}

[1] J S Carter, D Jelsovsky, S Kamada, L Langford, M Saito, Quandle cohomology and state-sum invariants of knotted curves and surfaces, Trans. Amer. Math. Soc. 355 (2003) 3947-3989 MR1990571

[2] J S Carter, S Kamada, M Saito, Geometric interpretations of quandle homology, J. Knot Theory Ramifications 10 (2001) 345-386 MR1825963

[3] R Fenn, C Rourke, Racks and links in codimension two, J. Knot Theory Ramifications 1 (1992) 343-406 MR1194995

[4] Y Ishii, A Yasuhara, Color invariant for spatial graphs, J. Knot Theory Ramifications 6 (1997) 319-325 MR1457191

[5] D Joyce, A classifying invariant of knots, the knot quandle, J. Pure Appl. Algebra 23 (1982) 37-65 MR638121 
[6] S Kamada, Knot invariants derived from quandles and racks, from: "Invariants of knots and 3-manifolds (Kyoto, 2001)", Geom. Topol. Monogr. 4, Geom. Topol. Publ., Coventry (2002) 103-117 MR2002606

[7] L H Kauffman, Invariants of graphs in three-space, Trans. Amer. Math. Soc. 311 (1989) 697-710 MR946218

[8] A Kawauchi, A survey of knot theory, Birkhäuser Verlag, Basel (1996) MR1417494 Translated and revised from the 1990 Japanese original by the author

[9] S Kinoshita, Alexander polynomials as isotopy invariants. I, Osaka Math. J. 10 (1958) 263-271 MR0102819

[10] S Kinoshita, Alexander polynomials as isotopy invariants. II, Osaka Math. J 11 (1959) 91-94 MR0110101

[11] F Luo, On Heegaard diagrams, Math. Res. Lett. 4 (1997) 365-373 MR1453066

[12] S V Matveev, Distributive groupoids in knot theory, Mat. Sb. (N.S.) 119(161) (1982) 78-88, 160 MR672410

[13] J McAtee, D S Silver, S G Williams, Coloring spatial graphs, J. Knot Theory Ramifications 10 (2001) 109-120 MR1822144

[14] T Mituhisa, Abstraction of symmetric transformations, Tôhoku Math. J. 49 (1943) 145-207 MR0021002

[15] E E Moise, Affine structures in 3-manifolds. V. The triangulation theorem and Hauptvermutung, Ann. of Math. (2) 56 (1952) 96-114 MR0048805

[16] S Satoh, Quandle cocycle invariants of knotted graphs, preprint

[17] S Suzuki, On linear graphs in 3-sphere, Osaka J. Math. 7 (1970) 375-396 MR0279799

[18] S Suzuki, Alexander ideals of graphs in the 3-sphere, Tokyo J. Math. 7 (1984) 233-247 MR752125

[19] F Waldhausen, Heegaard-Zerlegungen der 3-Sphäre, Topology 7 (1968) 195-203 MR0227992

[20] D N Yetter, Category theoretic representations of knotted graphs in $\mathbf{S}^{3}$, Adv. Math. 77 (1989) 137-155 MR1020582

Research Institute for Mathematical Sciences, Kyoto University

Kyoto 606-8502, Japan

aishii@kurims.kyoto-u.ac.jp

Received: 29 January 2008 Revised: 8 May 2008 